Revista

\title{
Multi-Ensayos
}

Vol. 8, núm. 15

ISSN: 2412-3285

https://multiensayos.unan.edu.ni

DoI: https://doi.org/10.5377/multiensayos.v8i15.13192

\section{Innovación en mercadeo: fortalecimiento de la competitividad de las Mipymes de la ciudad de Estelí}

\section{Innovation in marketing: strengthening the competitiveness of Mipymes in the city of Estelí}

Fabio José Gaitán Aguilera ${ }^{1}$

Recibido: 26 de agosto de 2021. Aceptado: 15 de diciembre de 2021

\section{RESUMEN}

En el presente ensayo se abordó la problemática que enfrenta la micro, pequeña y mediana empresa (Mipyme) en relación con la implementación de ciencia, tecnología e innovación (CTI) en sus procesos de comercialización de bienes y servicios. Este ensayo es de investigación sobre una temática de interés "Innovación en mercadeo", en él se presenta una conceptualización general, datos de estudios realizados en Mipymes de la ciudad de Estelí, que reflejan la necesidad de fortalecimiento en estrategias competitivas e innovación. La experiencia del autor en esta temática es tomada en cuenta y una propuesta de acciones para fortalecer la competitividad de estas empresas es presentada. La base metodológica consistió en la revisión bibliográfica de artículos científicos, tesis de grado y maestría relacionadas con fundamentos de CTI e innovación en mercadotecnia y de estudios a nivel nacional y local en diferentes Mipymes, para lo cual se consultaron bases de datos suscritas de la UNAN-Managua, el repositorio Centroamericano del CNU, revista científica de FAREM Estelí y Google académico entre otras. En los resultados de las investigaciones referenciadas, se evidencia la falta de: estudios del ambiente, estrategias de mercadeo, surtido de productos, posicionamiento en el mercado, diseño de marca, locales inadecuados, diferenciación de productos y servicios, marketing digital, deficiente atención al cliente, falta de organización gremial, entre otros. Se recomienda ante esta problemática acciones que fortalezcan sus procesos comerciales mediante innovaciones en mercadeo, seguir aunando esfuerzos mediante la articulación Estado, Empresas y Universidades identificando necesidades latentes y visualizando nuevas perspectivas de análisis de la problemática.

Palabras claves: innovación; fortalecimiento; competitividad.

1 Docente de la Facultad Regional Multidisciplinaria, Estelí. UNAN-Mangua/FAREM-Esteli. Correo electrónico: fgaitan3@yahoo. com. ORCID: https://orcid.org/0000-0002-6280-1087

(c) 2022 Revista Multi-Ensayos. 


\section{ABSTRACT}

This paper addresses the micro, small and medium-sized enterprises (Mipyme for its Spanish acronym) problems concerning the implementation of science, technology, and innovation (CTI) in their processes of marketing goods and services. This paper researches a topic of interest "Innovation in marketing." It presents a general conceptualization, data from studies carried out in different Mipymes in Estelí. These studies reflect the need for strengthening small business competitiveness, strategies, and innovation. The author's experience with this topic is taken into account to present a proposal for actions to strengthen the competitiveness of these companies. The methodology consisted of a bibliographic review of scientific articles, master and bachelor thesis on the foundations of CTI and innovation in marketing and studies at the national and local level in different Mipymes. The subscribed databases of the UNANManagua were consulted, for instance, the Central American repository of the CNU, scientific journal of FAREM Estelí, and Google Scholar among others. The results show the lack of environmental studies, marketing strategies, product assortment, market positioning, brand design, inadequate premises, product and service differentiation, digital marketing, poor customer care, lack of trade organization, among others. It is recommended to address actions to solve these problems to strengthen their business processes. Action can be done through innovations in marketing, continue to join efforts through the articulation of diverse entities such as the state, companies, and universities identifying latent needs and visualizing new perspectives of analysis of the problem.

Keywords: innovation; strengthening; competitiveness.

\section{INTRODUCCIÓN}

La micro, pequeña y mediana empresa (Mipyme) es uno de los sectores claves y de gran importancia en la economía de un país, forma parte como eslabón en el proceso de producción, distribución intercambio y consumo de bienes y servicios.

El auge económico, el incremento de la competencia, la exigencia cada día mayor que tienen los consumidores con respecto a los bienes y servicios que estos requieren, hacen necesario que los negocios tanto a nivel nacional como en la ciudad de Estelí visualicen de manera creativa y eficaz la manera en que van a atender a los consumidores.

La innovación en las empresas independientemente de su tamaño, se convierte en una necesidad imperativa para competir en un mundo globalizado y ayudar a países como el nuestro, Nicaragua, a encontrar nuevas vías para el crecimiento que no sean las tradicionales mediante la producción de bienes primarios (bienes destinados a satisfacer directamente necesidades de consumo), y el uso de los recursos naturales.

La innovación de mercadotecnia consiste en la aplicación de un nuevo método de comercialización realizando cambios en los diseños de productos y su envase, tratando de satisfacer mejor las necesidades de los consumidores, implica la introducción de un nuevo método de comercialización que antes no se utilizaba o una nueva estrategia que contribuya a abrir nuevos mercados o posicionarse de una manera diferente en el mercado para incrementar las ventas. (OCDE y eurostat, 2005) 
En general, la innovación en mercadotecnia comprende la generación de ideas innovadoras mediante cambios en los diseños de productos y nuevos métodos de comercialización.

Para las Mipymes en el ambiente actual es imprescindible identificar las debilidades en cuanto a innovación en mercadeo, para así implementar estrategias y acciones que fortalezcan su competitividad y logren un posicionamiento claro en el mercado.

Este ensayo se centra en los principales argumentos relacionados con la innovación en el mercadeo para el fortalecimiento de la competitividad de las Mipymes de la ciudad de Estelí.

En relación a este propósito, inicialmente en el desarrollo se presenta la conceptualización e importancia de la innovación en mercadotecnia, de manera continua en base a investigaciones realizadas, se evidencia las debilidades existentes en diversos sectores de las Mipymes en cuanto a innovación en mercadeo, lo cual comprende: el análisis del ambiente empresarial, estrategias en la mezcla de marketing, el uso de tecnologías y el servicio al cliente.

De manera adicional se expone la experiencia del autor de este escrito en el sector de ropa y calzado de las Mipymes y en el sector educativo universitario.

Se concluye con una propuesta de sugerencias de acciones de innovación en mercadeo de autoría propia, enfocadas en fortalecer la competitividad de las Mipymes que permitan brindar un mejor producto o servicio a los clientes y por ende incrementar sus beneficios económicos.

\section{DESARROLLO}

\section{Conceptualización e importancia de la Innovación en las empresas}

El ambiente empresarial actual está caracterizado por una constante lucha por obtener un mayor reconocimiento por sus clientes y lograr más posicionamiento en el mercado respecto a sus competidores. La innovación es esencial para la competitividad de las empresas, de manera específica en el proceso de gestión comercial que estas implementan. Su importancia aumenta debido a la globalización de los mercados, los ciclos más cortos de la vida de los productos y los cambios rápidos en los gustos y preferencias de los consumidores.

La Organización de Cooperación y Desarrollo Económicos (ODCE) (2005) (a como se citó en Amores (2015)) define la innovación como: "la implementación de un producto (bien o servicio) nuevo o significativamente mejorado, o proceso, o método de marketing o mejora organizativa en las prácticas empresariales, en la organización del lugar de trabajo o las relaciones externas". (pág. 105)

De igual manera La Organización de Cooperación y Desarrollo Económicos (ODCE) (2005) indica que: 
La innovación tiene como propósito la mejora de los resultados de la empresa mediante la obtención de ventajas competitivas incrementando la demanda de los productos, la reducción de los costes de transacción, o mejorando la capacidad de la empresa para innovar al acceder a nuevos conocimientos o crearlos. (pág. 44)

Lo antes expuesto, indica la necesidad de innovación en las empresas que les permita definir estrategias enmarcadas en mejorar la capacidad competitiva en base a sus costos, innovación y generación y apropiación de nuevos conocimientos que les permita tener ventajas competitivas en sus productos o servicios.

La innovación se puede clasificar de cuatro maneras: Innovaciones de productos, innovaciones de procesos, innovaciones organizativas e innovaciones de mercadotecnia. Para los fines abordados en este escrito se hará referencia a las innovaciones de mercadotecnia.

Las acciones de innovación que se emprendan en el área de mercadotecnia son determinantes en la fidelidad de los clientes y la forma en que se posicione en estos los productos y servicios ofertados.

Las innovaciones en mercadotecnia implican la puesta en práctica de nuevos métodos de comercialización. Estos pueden incluir cambios en el diseño y envasado de los productos, en la promoción y colocación de los productos y en los métodos de tarificación de los bienes y servicios. (OCDE y eurostat, 2005, pág. 24)

Desarrollar de manera constante innovaciones en mercadotecnia es una necesidad imperativa para las empresas si quieren sobrevivir y tener beneficios económicos aceptables de acuerdo a las inversiones realizadas.

Gómez, (2008), (a como se citó en Ospina, Puche y Arango (2014)) indica la necesidad de la innovación en las Mipymes al referir que:

Debido a la gran oferta que hay en el mercado de las pequeñas y medianas empresas, estas se han visto obligadas a implementar ideas innovadoras y eficientes que les permitan posicionarse y ser competitivas dentro del mismo. Esta innovación se logra modificando sus estrategias, desarrollando nuevos productos, nuevos canales de distribución, nuevos métodos de comercialización y nuevos procesos de producción. (pág. 59)

\section{Problemas que enfrentan las empresas en innovación de mercadeo}

Desde el punto de vista de la gestión comercial, las empresas presentan el problema de que se enfocan solamente en crear o mejorar los productos existentes sin darle relevancia a otros aspectos de la gestión comercial.

En un estudio realizado a nivel nacional en un grupo de 26 empresas en Nicaragua, la práctica de la innovación se limita casi totalmente a la creación de nuevos productos o a la mejora de productos existentes, así como a la mejora del proceso de producción, es decir, a las innovaciones de tipo técnico. 
Muy pocas han identificado el gran potencial de las innovaciones de tipo administrativo u organizativo como prácticas laborales y modelos de negocios. (Escobar Cerda, 2009)

A nivel local en la ciudad de Estelí se han realizado diversos estudios en distintos tipos de Mipymes, en las que se evidencia los problemas que tienen en la comercialización de sus productos, debido a la carencia de innovación en mercadeo. A continuación, se presentan algunos sectores en los que se han realizado estudios y que se muestra la problemática en mención:

En el sector comercio de ropa y calzado existe poca publicidad y promociones, débil surtido de productos, locales pequeños y no muy presentables, no tienen mucha diferenciación de los productos, la mayoría no utiliza tecnologías de la información y las comunicaciones (TIC) como ser tarjetas de crédito, marketing digital, tienen deficiente atención al cliente, débil posicionamiento en el mercado, entre otras, que inciden en el posicionamiento, las ventas y la fidelidad de sus clientes (Gaitán Aguilera, 2016).

En las empresas de cuero y calzado se realizó un estudio a 12 pymes de la ciudad de Estelí, en las cuales se refleja los altos costos de la materia prima, la saturación de la oferta en el mercado, el estudio refleja que estas empresas no cuentan con conocimientos necesarios en cuanto a mercadeo se refiere, ni con una organización gremial, los locales son pequeños y mal organizados; se carece de registros contables; la mayoría de las empresas necesitan establecer una marca y no se invierte en publicidad. (Diaz Pastrana, 2012)

En el sector lácteo se observa la falta de innovación que incide en la gestión comercial de sus productos estando entre estas: empresas enfocadas en el mercado local que tienen poca exigencia en calidad y esto no permite mejorar la competitividad y mejorar los ingresos, la producción de derivados de la leche se baja en temporada de verano, su capacidad productiva y tecnológica es muy artesanal y es poca competitiva con respecto a los productos industrializados tanto nacionales como locales. Adicionalmente no tienen estrategias de crecimiento o mejora tecnológica para el futuro lo cual limita su desarrollo y crecimiento. (Briones Amador, Quezada Gómez, Matamoros Cornejo, \& Castillo Herrera, 2015)

En el sector ferretero se refleja las dificultades en el uso de herramientas de mercadeo para ofertar sus productos, a los cuales les falta mejorar en publicidad y herramientas para promocionar los productos, la utilización de más medios para dar a conocer los productos a los clientes, promociones e innovación de artículos en el inventario lo cual representa debilidades que afectan su competitividad en el mercado. (Ruiz Castro, Peralta Merlo, \& López Iglesias, 2020)

Las Mipymes del sector panadero enfrentan debilidades siendo entre estas: carecer de conocimientos técnicos y experiencia para identificar oportunidades y amenazas que influyen en su participación en el mercado; enfrentarse a un mercado altamente competitivo y en ocasión desleal. Esto incide en las decisiones y estrategias que como empresa debe formular para competir en el mercado. (Fortín Altamirano, Rodríguez Ruiz, \& Blandón Pérez, 2019).

La industria de agua embotellada, refiere que no ha logrado posicionarse en el mercado, porque no ha desarrollado una estrategia de mercado de manera eficiente y no ha identificado otras estrategias 
de marketing adecuadas que contribuyan al posicionamiento (Centeno Cruz, Espinoza Hudiel, \& López Briones, 2014).

Al analizar la situación de las Mipymes de los distintos sectores en los estudios antes descritos, las oportunidades de mejora se evidencia en el fortalecimiento de su competitividad en los siguientes aspectos: estudio del ambiente y factores que inciden en la empresa, estrategias de mercadeo en cuanto a actividades promocionales, surtido de productos, posicionamiento en el mercado, marca para sus productos, condiciones de los locales, diferenciación de los productos y servicios, uso de TIC como son tarjetas de crédito en línea, marketing digital, fortalecer el área de atención al cliente, irregularidad en el abastecimiento de materia prima, entre otros.

Como experiencia personal como autor de este ensayo, he estado inmerso durante más de 25 años (19902015) en el comercio detallista de ropa y calzado en la ciudad de Estelí, durante el cual he evidenciado y compartido experiencia con comerciantes sobre los distintos problemas a los cuales nos enfrentábamos, siendo estos en parte los reflejados en los estudios antes mencionados. Muchas de estas debilidades han estado marcadas por la falta de recursos económicos, por la falta de estudios más detallados del mercado, implementación de estrategias competitivas y por la inexistencia en años anteriores de medios sociales digitales que resultan más económicos para posicionarse y dar a conocer los productos en el mercado.

De igual manera, he acumulado experiencia en el ejercicio de la docencia en el área administrativa y de mercadeo, así como, en la realización de investigaciones dirigidas al sector comercio específicamente, en las estrategias de comercialización del sector de ropa y calzado de la ciudad de Estelí.

En base a experiencias personales, se sugiere a continuación a los propietarios y gerentes algunas medidas que se han comprobado exitosas para mejorar la competitividad en las Mipymes según convenga el área de negocios en la cual estén inmersos.

\section{Propuesta de innovación para fortalecer la competitividad en las Mipymes}

En relación con la problemática planteada en los estudios en mención se hace necesario fortalecer la competitividad y el posicionamiento de las mipymes en los siguientes aspectos:

- Realizar un análisis del ambiente empresarial utilizando la herramienta FODA (Fortalezas, oportunidades, debilidades y amenazas)

- Segmentar el mercado y elegir el mercado meta al cual va a dirigir su oferta de productos, acompañado de acciones de mercadotecnia que le dé una mayor atracción y presentación de sus negocios.

- Diversificación en líneas de productos y estrategias claras de posicionamiento acompañada de acciones innovadoras para crear lealtad de los clientes.

- Implementar nuevas estrategias de penetración de nuevos mercados.

- Aprovechamiento de las TIC para brindar mejor servicio y tener mayor contacto con sus clientes.

- Realización de alianzas con competidores que ofrezcan productos complementarios.

- Agremiarse para unificar esfuerzos y lograr sinergia. 
- Monitorear constantemente la opinión de los consumidores para implementar acciones que permitan diferenciar sus negocios de los competidores y la creación de clientes fieles.

- Diversificar sus negocios.

- Establecer alianzas con Universidades que les brinden conocimientos científicos en sus respectivos sectores, así como aprovechar las distintas formas de apoyo brindadas por las instituciones del Estado enfocadas en el apoyo a las Mipymes.

- Capacitar de manera constante a sus trabajadores para brindar un buen servicio y lograr un desarrollo organizacional.

- Invertir en equipos tecnológicos para mejorar la producción y calidad de los productos.

Es importante señalar la existencia de lineamientos, estrategias y políticas concretas implementadas por el Gobierno de Unidad y Reconciliación Nacional se derivan del Plan Nacional de Desarrollo Humano (PNDH, 2018-2021) dirigidas a mejorar la competitividad de las Mipymes en Nicaragua.

Para este fin, están involucradas diferentes instituciones del Gobierno Central, mediante diferentes programas de apoyo como son: Consejo Nicaragüense de Ciencia y Tecnología (CONICYT), Ministerio de Fomento, Industria y Comercio (MIFIC), Instituto Nicaragüense de Fomento Municipal (INIFOM), Ministerio de la Economía Familiar, Comunitaria, Cooperativa y Asociativa (MEFCCA), Instituto Nicaragüense de Apoyo a la Pequeña y Mediana Empresa (INPYME), gobiernos locales a través de las Alcaldías, de igual manera Universidades y Centros de Investigación, Organismos de corte Sectorial y los propietarios de las Mipymes, que de manera articulada están trabajando para fortalecer las capacidades de los distintos sectores de la economía.

\section{CONCLUSIONES}

El ambiente competitivo actual exige a las empresas llevar a cabo transformaciones mediante la introducción de nuevas actividades de mayor valor añadido. La ciencia, tecnología e innovación (CTI) es fundamental para el desarrollo económico y el progreso tanto a nivel empresarial como de la sociedad en general; inmersa en este proceso la innovación, tanto a nivel macro como a nivel micro, se vuelve de gran importancia para competir en un mundo globalizado.

La innovación es un proceso continuo presente en cualquier sector de la economía, por lo que las empresas de forma permanente deben efectuar cambios en los productos, en los procesos y captar nuevos conocimientos; este proceso no se debe limitar estrictamente a las empresas emergentes, sino también a las ya existentes en el mercado.

La competitividad en las Mipymes es un elemento de mucha importancia, ya que, se necesita contar con conocimientos, tecnología y procesos innovadores que le permitan su continuidad en el mercado para lograr los beneficios esperados.

En Nicaragua y de manera particular en la ciudad de Estelí, de acuerdo con las investigaciones reflejadas en este ensayo, es evidente la debilidad o carencia en algunos negocios, en cuanto a estrategias, acciones, 
procesos de innovación en mercadotecnia, lo que ha afectado de manera directa su capacidad competitiva ocasionando el fracaso y en el mejor de los casos han logrado mantenerse en el punto de equilibrio.

Por tal razón, se hace necesario fortalecer aún más la capacidad competitiva de las Mipymes, dotándolas de todos los elementos mercadológicos necesarios e innovadores en sus procesos de comercialización mediante la introducción de un nuevo método que antes no se utilizaba o una nueva estrategia que contribuya a abrir nuevos mercados o posicionarse de una manera diferente para incrementar las ventas. . Para esto es pertinente continuar a como se está realizando, con la articulación entre Estado, Empresas y Universidades, de modo que se identifique las necesidades latentes que contribuyan a mejorar la posición competitiva de sus negocios y visualizar nuevas perspectivas de análisis de la problemática de cara a los cambios del ambiente competitivo global. Bajo esta premisa, en este ensayo se ha realizado propuestas de manera general de elementos básicos que contribuyan a mejorar la competitividad de las Mipymes.

\section{REFERENCIAS}

Amores Bravo, X. (2015). La gestión de la innovación en las empresas de servicios intensivas en conocimiento tecnológico (t-KIBS) de Cataluña. Recuperado el 17 de Junio de 2021, de https://www.tdx.cat/ handle/10803/380897\#page $=1$

Briones Amador, G. A., Quezada Gómez, J. E., Matamoros Cornejo, M. M., \& Castillo Herrera, B. (2015). Competitividad de las MIPYMES que se dedican a la producción y comercialización de productos lácteos, 2014. Revista Cientifica de FAREM- Esteli(15), 45-53. Obtenido de https://repositorio.unan. edu.ni/6326/1/215-802-1-PB.pdf

Centeno Cruz, J. V., Espinoza Hudiel, S. L., \& López Briones, D. J. (2014). Importancia de la implementación de estrategias de marketing en el posicionamiento de empresa Agua Roca en la ciudad de Estelí, en el año 2014. Obtenido de https://repositorio.unan.edu.ni/1659/1/16341.pdf

Diaz Pastrana, J. R. (Abril de 2012). Producción y comercialización de 12 PYMES de cuero y calzado legalmente inscritas en la Alcaldía Municipal de la ciudad de Estelí. 2009-2011. Revista Cientifica de FAREM- Esteli(1), 28-30. Obtenido de https://repositorio.unan.edu.ni/5776/2/7-20-1-PB.pdf

Escobar Cerda, O. A. (2009). Gerencia de la Innovación en Pymes de Nicaragua (Estudio de campo en 26 Pymes de Nicaragua). Encuentro(82), 39-54. Recuperado el 17 de junio de 2021, de https://core. ac.uk/download/pdf/267025465.pdf

Fortín Altamirano, R., Rodríguez Ruiz, I., \& Blandón Pérez, N. (1 de Julio de 2019). Proceso administrativo de Microempresa Panadería El Esfuerzo y sus efectos socioeconómicos en las familias de las beneficiadas del Programa Hambre Cero en la comunidad Los Jobos, Estelí, 2017-2018. Obtenido de https:// repositorio.unan.edu.ni/11580/1/20124.pdf

Gaitán Aguilera, F. J. (2016). Estrategias competitivas de comercialización utilizadas por las tiendas de ropa y calzado del distrito I, de la ciudad de Estelí, año 2014. Recuperado el 17 de junio de 2021, de https://repositorio.unan.edu.ni/2817/

OCDE y eurostat. (2005). Manual de Oslo. Recuperado el 17 de junio de 2021, de http://www.itq.edu.mx/ convocatorias/manualdeoslo.pdf

Ospina Zapata, M., Puche Nieves, M. A., \& Arango Alzate, B. (19 de mayo de 2014). Gestión de la Innovación en Pequeñas y Medianas Empresas. Generando ventajas competitivas y posicionamiento en el 
Mercado. Gestión de las personas y tecnología.(19), 56-67. Obtenido de https://www.redalyc.org/ articulo.oa?id=477847107005

Ruiz Castro, K. S., Peralta Merlo, G. F., \& López Iglesias, F. Y. (2020). Estrategias de comercialización de la empresa familiar CONSTRUNICA con respecto a empresas similares de la ciudad de Estelí, periodo 2018-2019. Recuperado el 17 de junio de 2021, de https://repositorio.unan.edu.ni/13239/1/19960. pdf

Suárez González, A. (2011). Modelo para la gestión de comercialización en la empresa comercializadora del SIME DIVEP VC. (U. C. VILLAS, Ed.) Recuperado el marzo de 2021, de https://dspace. uclv.edu.cu/bitstream/handle/123456789/3652/ANABEL\%20SUAREZ\%20GONZALEZ. pdf?sequence $=1$ \&isAllowed $=y$ 